\title{
Precisión y eficiencia del inventario de plantaciones de teca en Ecuador mediante escáner láser terrestre
}

\section{Precision and efficiency of the inventory of teak plantations in Ecuador by using terrestrial laser scanner}

\author{
Alberto Peñalver Romeo', Fernando J. Aguilar Torres², Abderrahim Nemmaoui'2, José Ricardo Rivas Barzola', \\ Ángel Antonio Triana Tomalá', Manuel A. Aguilar Torres² y Alfonso Llanderal'
}

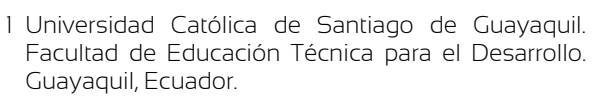

2 Universidad de Almería. Departamento de Ingeniería.
Almería, España.

\section{RESUMEN}

El objetivo de la investigación fue evaluar la precisión y eficiencia del método de inventario forestal usando el escáner láser terrestre para la determinación de variables dendrométricas en plantaciones de teca (Tectona grandis Linn. F.). Se establecieron sitios de muestreo circulares de $500 \mathrm{~m}^{2}$ en plantaciones de teca de tres predios de la región Costa de Ecuador donde se usaron las metodologías de inventario forestal tradicional y del escáner láser terrestre, registrando las variables dendrométricas (diámetro normal, altura total y radio de copa) y el tiempo de trabajo necesario para complementar el inventario de cada parcela. Las estimaciones de los índices dasométricos para cada método de inventario y la eficiencia relativa del método del escáner láser terrestre con respecto al inventario forestal tradicional fueron contrastadas mediante pruebas de inferencia estadística. La prueba de contrastes estadísticos aplicada no detectó diferencias significativas entre los dos métodos de inventario en relación tanto con las variables dasométricas medidas como con los tiempos de trabajo registrados en cada parcela. Los valores de eficiencia relativa evidenciaron cierta superioridad del inventario realizado con el escáner láser terrestre sobre el realizado con el inventario forestal tradicional. El presente estudio permite concluir que el método del escáner láser terrestre para la elaboración de inventarios forestales en plantaciones de teca es preciso para la estimación de variables dasométricas, siendo más eficiente que el de inventario tradicional.

Palabras ClaVe: Costa de Ecuador, dendrometría, inventario forestal, Tectona grandis.

\section{ABSTRACT}

The aim of this study was to evaluate the precision and efficiency of the forest inventory method from using Terrestrial Laser Scanner (TLS) to determine dendrometric variables in teak plantations (Tectona grandis Linn. F.). Circular sampling plots of $500 \mathrm{~m}^{2}$ were established in three teak plantations located in the Coastal region of Ecuador. Traditional forest inventory (TFI) and TLS methods were used to determine several dendrometric variables (normal diameter, total height, and crown radius), also recording the work time necessary to complement the inventory of each plot. The estimates of the dasometric indices for each inventory method and the relative efficiency of TLS with respect to IFT were contrasted using statistical inference tests. The statistical tests did not detect significant differences between the two inventory methods in relation to both the measured dasometric variables and the working times recorded in each plot. The relative efficiency values showed some superiority of TLS method over TFI. Forest inventories of teak plantations based on TLS method is sufficiently accurate for estimating dasometric variables, being more efficient than traditional inventory.

KEYWORDS: Coastal region of Ecuador, dendrometry, forest inventory, Tectona grandis. 


\section{INTRODUCCIÓN}

Los inventarios forestales permiten obtener información de los bosques naturales y plantaciones para planificar su manejo y explotación. En la actualidad, se incluyen otros objetivos adicionales relacionados con aspectos como la biodiversidad, la producción de biomasa y el secuestro de carbono atmosférico. Es importante destacar que existe la necesidad de información global para el desarrollo de modelos de manejo de estos ecosistemas, teniendo en cuenta los efectos del cambio global, la desertificación, la producción de agua y sus interacciones con la biosfera (Bahamondez, Lorenz, Mery y Varjo, 2005).

Para hacer frente a las necesidades de información sobre superficies, crecimiento y rendimiento de las plantaciones forestales se requiere incrementar la eficacia y eficiencia en la realización de sus inventarios (Suraj Reddy, Rakesh, Jha y Rajan, 2018). En este sentido, constituye una prioridad la evaluación de las tecnologías de captura de datos geoespaciales basadas en la teledetección mediante plataformas satelitales, aéreas y terrestres (White et al., 2016). En efecto, el uso de sensores remotos, como la tecnología Light Detection and Ranging (LiDAR) mediante el uso del Terrestrial Laser Scanner (escáner láser terrestre; TLS por sus siglas en inglés) o Airborne Laser Scanner (escáner láser aéreo), la estereofotogrametría aerotransportada de alta resolución o las imágenes satelitales multiespectrales (Kershaw, Ducey, Beers y Husch, 2017; White, et al., 2016) son los pilares de una disciplina emergente denominada Silvicultura de Precisión (Bare, 2003). El objetivo de esta disciplina es desarrollar técnicas y procedimientos para aumentar la precisión de la extracción de datos forestales, así como incrementar la cantidad y calidad de los datos disponibles para mejorar la toma de decisiones en gestión forestal económica y medioambientalmente sostenibles (Bare, 2003).

El rápido desarrollo de los dispositivos TLS en los últimos años ha despertado el interés en relación con su aplicabilidad para la ejecución de inventarios forestales, especialmente cuando las mediciones directas son muy caras o casi imposibles de realizar con las metodologías propias del inventario forestal tradicional (IFT), independientemente de que los instrumentos TLS se venden a precios que pueden hacer prohibitivo su uso práctico y requieren de personal técnico altamente especializado para su operación (Abegg, Kükenbrink, Zell, Schaepman y Morsdorf, 2017). Sin embargo, todavía se necesitan estudios exhaustivos dirigidos a la evaluación de su precisión y eficiencia en relación con los métodos de inventario tradicional (Abegg et al., 2017).

El TLS es un instrumento capaz de obtener información topográfica y geométrica de una estructura de manera precisa, así como de capturar la información radiométrica de los objetos (Porras Díaz, Cáceres Jiménez y Gallo Lancheros, 2014). Esto puede ser posible dada la naturaleza 3D de la nube de puntos obtenida por medio del TLS (Liang et al., 2016). Cabe destacar que no existen dos nubes de puntos iguales de una misma superficie tomadas con el mismo aparato y desde la misma posición (Ramos, Marchamalo, Rejas y Martínez, 2015).

\section{OBJETIVOS}

El objetivo de la investigación fue evaluar la precisión y eficiencia del método de inventario forestal mediante TLS para la determinación de variables dendrométricas en plantaciones de teca (Tectona grandis Linn. f.) de la Región Costa de Ecuador.

\section{MATERIALES Y MÉTODOS}

La población objeto de estudio está constituida por tres plantaciones de teca ubicadas en la provincia de Guayas, Región Costa de Ecuador. Las áreas de trabajo se sitúan en las plantaciones de Morondava $\left(2^{\circ} 6^{\prime} 11.72^{\prime \prime} \mathrm{S}\right.$, $\left.80^{\circ} 2^{\prime} 59.43^{\prime \prime W}\right)$, El Tecal (1³1'53.07"S, 80²'30.51"W) y Allteak (18 $\left.8^{\prime} 7.23^{\prime \prime} \mathrm{S}, 79^{\circ} 41^{\prime} 58.08^{\prime \prime W}\right)$. Las características de cada plantación se presentan en la tabla 1. 
TABla 1. Descripción de las plantaciones de teca (Tectona grandis Linn. F.) en la provincia de Guayas, Región Costa de Ecuador, en las que se evaluó la precisión y eficiencia del método de inventario forestal mediante la utilización del escáner laser terrestre.

\begin{tabular}{ccccccccccccc}
\hline Predio & Área (ha) & $\mathrm{n}$ & $\mathrm{A}$ & $\mathrm{NI}$ & $\mathrm{Na}$ & $\mathrm{dg}$ & $\mathrm{G}$ & $\mathrm{H}$ & $\mathrm{hL}$ & $\mathrm{hdom}$ & $\mathrm{E} \%$ & $\mathrm{~V}$ \\
\hline Morondava & 78.3 & 31 & 2.9 & 825 & 703 & 7.5 & 3.9 & 6.9 & 7.6 & 9.2 & 58.9 & 21.1 \\
Tecal & 21.1 & 8 & 17.0 & 1373 & 985 & 12.5 & 16.8 & 14.0 & 15.4 & 19.9 & 144.7 & 122.3 \\
All Teak & 57.2 & 23 & 10.5 & 1003 & 595 & 12.0 & 11.4 & 12.5 & 18.8 & 21.3 & 94.1 & 85.9 \\
\hline
\end{tabular}

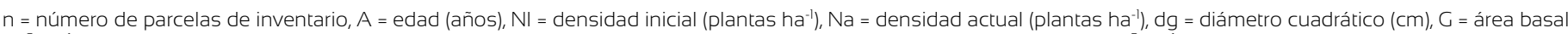
$\left(\mathrm{m}^{2} \mathrm{ha}^{-1}\right), \mathrm{hL}=$ altura lorey $(\mathrm{m})$, hdom = altura dominante $(\mathrm{m}), \mathrm{hc}=$ altura comercial $(\mathrm{m}), \mathrm{E}=$ espesura $(\%), \mathrm{V}=$ volumen total $\left(\mathrm{m}^{3} \mathrm{ha}^{-1}\right)$.

El clima de la región donde se ubican las plantaciones se caracteriza por una precipitación promedio de aproximadamente $1222 \mathrm{~mm}$, con una temperatura media de 24.4 ${ }^{\circ} \mathrm{C}$ y una humedad relativa de $72.9 \%$ (Flores Velasteguí, Cabezas Guerrero, Crespo Gutiérrez, 2010). La zona en la que se realizó el estudio pertenece al llamado bosque seco tropical (Holdridge, 1982), que se caracteriza por un régimen de lluvia unimodal muy típico con un período de lluvia en el primer trimestre del año y una marcada sequía durante el resto del año. Estas condiciones climáticas provocan una fenología forestal estacional, presentando temporadas alternas de follaje (de enero a julio) y sin follaje (de septiembre a diciembre).

Para lograr los objetivos de este trabajo, se realizaron comparaciones de precisión y eficiencia a escala de parcela entre el inventario TLS y el IFT. También se evaluó la precisión de cada método a escala de árbol y el efecto de la reducción del tamaño de la parcela TLS en la precisión de la estimación de las variables dasométricas.

\section{Metodología del inventario forestal tradicional}

Mediante un diseño probabilístico estratificado (plantación) se seleccionaron 62 parcelas de forma circular de $500 \mathrm{~m}^{2}$ (radio de $12.62 \mathrm{~m}$ ) y se distribuyeron en cada plantación (estratos) de manera aleatoria (Tabla 1). Cabe destacar que el número de parcelas en cada plantación fue proporcional a su superficie según una intensidad de muestreo de $2 \%$. La recolección de datos se realizó durante los meses de marzo a mayo de 2018 (con hojas en el árbol de teca).

En cada parcela se midieron la circunferencia normal de cada árbol (circunferencia a la altura del pecho; CAP) y los cuatro radios de la proyección horizontal de la copa de cada árbol sobre el suelo (direcciones norte, este, sur y oeste). Esta última fue determinada con brújula magnética, midiendo la proyección horizontal de la copa con una cinta métrica (estas mediciones directas con cinta se suponen libres de errores instrumentales). Finalmente, se midió la altura total de cada árbol con un hipsómetro láser telémetro LaserAce ${ }^{\mathrm{TM}}$ 1000, aplicando los procedimientos de medición y registro de datos propios de la dasometría (Kershaw, Ducey, Beers y Husck, 2017). Igualmente, se anotaron los tiempos de trabajo para el establecimiento de la unidad de muestreo y la medición de árboles.

\section{Metodología del inventario digital mediante TLS}

La toma de datos para el inventario TLS se realizó en noviembre del 2018 (6 meses después del IFT). Cabe destacar que las plantaciones se encontraban en un estadio fenológico sin hojas. Las mediciones se realizaron usando el TLS FARO Focus 3D X330 (Aguilar, Nemmaoui, Peñalver, Rivas y Aguilar, 2019a). Este equipo es capaz de capturar millones de puntos $3 \mathrm{D}$ con una precisión de distancia nominal de alrededor de $\pm 2 \mathrm{~mm}$ a lo largo de un intervalo de $0.6 \mathrm{~m}$ a $330 \mathrm{~m}$ y campos de visión vertical y horizontal de $300^{\circ}$ y $360^{\circ}$, respectivamente. Los parámetros óptimos de calidad y tiempo se realizaron de acuerdo con lo propuesto por Aguilar et al. (2019a). Se tomaron imágenes RGB panorámicas mediante la propia cámara de 70 Megapixeles que incorpora el instrumento. Estas imágenes se emplearon para colorear la nube de puntos 3D y como referencia visual de alta calidad en las tareas de postprocesamiento en gabinete. Todos los sensores que 
incorpora el escáner fueron activados (i.e., GPS, inclinómetro, brújula y altímetro).

El equipo se ubicó en el centro de las parcelas de referencia del inventario tradicional. Se establecieron cuatro posiciones de escaneo dentro de un radio de $18 \mathrm{~m}$ (representa una superficie de $1017.87 \mathrm{~m}^{2}$, lo que duplica el área de muestreo del IFT), para configurar un patrón de escaneo con un escáner central y los otros alrededor, dibujando un triángulo equilátero. Cabe destacar que los 4 puntos de escaneo se georreferenciaron con la ayuda de nueve esferas (de $15 \mathrm{~cm}$ de diámetro y color blanco) convenientemente distribuidas en la parcela (siendo visibles cada dos escaneos consecutivos) (Aguilar, Rivas, Nemmaoui, Peñalver y Aguilar, 2019b). Estas esferas son detectadas automáticamente en la nube de puntos mediante el software Scene ${ }^{\mathrm{TM}}$ versión 7.1 (Scene, 2017). Las coordenadas de las esferas se midieron con una estación total TOPCON ES105 orientada mediante tres bases localizadas en campo abierto y previamente georreferenciadas mediante técnicas GPS-RTK (Aguilar et al., 2019b).

A partir de la nube de puntos muy densa obtenida en cada parcela, se llevó a cabo un proceso de segmentación de cada árbol para la extracción de variables dendrométricas (diámetro normal, altura total y radio de copa) mediante el uso del software libre 3D Forest (Trochta, Krůček, Vrška y Král, 2017).

\section{Comparación de los métodos IFT y TLS a escala de parcela y de árbol}

Las variables seleccionadas para la comparación de los métodos de inventario TLS e IFT a escala de parcela de muestreo fueron los valores promedio por parcela del diámetro a la altura del pecho (DAP) $(\mathrm{cm})$, altura total $(\mathrm{H})$ (m) y radio de copa (m). Para la evaluación a escala de árbol se compararon las variables dendrométricas extraídas de un conjunto de árboles (población muestral) pertenecientes a parcelas de las tres plantaciones estudiadas (Tabla 1). Los valores para la comparación fueron obtenidos a partir de los registros de campo del IFT y de mediciones realizadas sobre las nubes de puntos TLS. Es importante destacar que estas son las variables dendrométricas más comunes, debido a la posibilidad de su medición instrumental directa en el contexto del inventario forestal tradicional. Los árboles con un $\mathrm{DAP} \leq 5 \mathrm{~cm}$ fueron excluidos de la muestra en ambos inventarios.

\section{Evaluación de la precisión de los inventarios IFT y TLS a escala de parcela}

A fin de contrastar la precisión de los métodos de inventarios IFT y TLS, se calcularon los valores promedio de DAP (medido a $1.3 \mathrm{~m}$ ), altura total y radio de copa en cada parcela y para cada tipo de inventario. Las comparaciones se hicieron para cada plantación por separado. Para el contraste de las precisiones se aplicó la prueba t Student para muestras independientes $(\mathrm{p}<0.05)$ mediante el paquete estadístico INFOSTAT (2010). Se realizaron las pruebas de contraste de las diferencias de medias entre las variables objeto de estudio para los métodos de inventario evaluados, suponiendo una completa aleatorización de las unidades de muestreo.

\section{Evaluación de la eficiencia de los inventarios IFT y TLS a escala de parcela}

Para la evaluación de la eficiencia relativa del método de inventario mediante TLS (parcelas de radio $18 \mathrm{~m}$ ) versus parcelas de inventario forestal tradicional de tamaño $500 \mathrm{~m}^{2}$ se utilizó el procedimiento descrito por Kershaw et al. (2017). La ecuación 1 estima la eficiencia (e) en el inventario de la unidad de muestreo 2 en relación con la eficiencia en la unidad de muestreo 1 (muestreo de referencia). Si e $>1$ indica que la unidad de muestreo dos se llevó a cabo de una forma más eficiente que la unidad de muestreo uno y viceversa.

$$
e=\frac{\left(S_{x 1}\right)^{2} t_{1}}{\left(S_{x 2}\right)^{2} t_{2}}
$$

Donde:

$S_{X 1}=$ error estándar (en porcentaje) para una unidad de muestreo (parcela) como base de comparación 
$S_{X 2}=$ error estándar (en porcentaje) para la unidad de muestreo a comparar

$\mathrm{t}_{1}=$ tiempo para llevar a cabo la unidad de muestreo de referencia

$\mathrm{t}_{2}=$ tiempo para llevar a cabo la unidad de muestreo comparada

Los valores de tiempo fueron obtenidos a partir de los registros de mediciones en campo para ambos tipos de inventario. Adicionalmente, se realizó una prueba t Student $(\mathrm{p}<0.05)$ para contrastar los tiempos de trabajo en campo entre los dos métodos probados.

\section{Evaluación de la reducción del tamaño de parcela en TLS}

Para evaluar el efecto del tamaño de la parcela de medición mediante TLS en la precisión de las variables dendrométricas se aplicó un recorte a la parcela original TLS de $1018 \mathrm{~m}^{2}$ (radio de $18 \mathrm{~m}$ ) para reducirla al tamaño de la parcela de inventario tradicional de $500 \mathrm{~m}^{2}$ (radio de $12.62 \mathrm{~m})$.

Se copiaron los datos de la parcela original TLS en el tabulador de datos Excel®, incluyendo el valor de la distancia desde el centro de la parcela a cada árbol calculada a partir de las coordenadas de la base de cada árbol segmentado con el software 3D Forest. Se usó una fórmula condicional de la biblioteca Excel para eliminar todos los árboles que estuviesen a una distancia del centro de la parcela mayor a $12.62 \mathrm{~m}$. Con la información dendrométrica de los árboles de la parcela "recortada" se calcularon los valores promedio de diámetro normal, altura total y radio de copa. Se aplico la prueba t Student $(\mathrm{p}<$ 0.05) para muestras independientes como se mencionó previamente.

\section{Evaluación de la precisión de los inventarios IFT y TLS a escala de árbol}

Para la comparación de la precisión a nivel de árbol se procedió al "case de árboles", trabajo que se refiere a la identificación y emparejamiento de los árboles medidos en el IFT y los segmentados por el software 3D Forest a partir de la nube de puntos TLS en cada parcela.

El procedimiento empleado para el "case de árboles" fue el siguiente:

1. Identificación de los árboles numerados en el inventario terrestre en los escaneos georreferenciados (WGS84 UTM Zona 17S) del inventario digital (TLS) y extracción de las coordenadas XY de cada árbol. Esta acción se realizó en el entorno del software Scene versión 7.1 (Scene, 2017) aprovechando las imágenes panorámicas RGB ofrecidas por el TLS Faro Focus 3D. Las coordenadas de todos los árboles identificados en cada parcela se almacenaron en formato vectorial *shp.

2. Elaboración de un fichero de puntos (*shp) para cada parcela correspondientes a la posición de cada árbol extraído de la nube de puntos TLS mediante el software 3D Forest.

3. Agrupamiento de las variables dendrométricas de cada árbol obtenidas a partir de inventario tradicional y TLS mediante la unión de los dos ficheros *shp, generados en los dos pasos indicados anteriormente. Para ello se empleó el software ArcGis versión 10.3 (ArcGis, 2014) (https://desktop.arcgis.com/en/), estableciendo una condición de distancia máxima entre árboles de $60 \mathrm{~cm}$.

4. Una vez unidos los datos, se procedió a su revisión y verificación, almacenándose en formato Excel para facilitar su análisis conjunto.

5. Para evaluar si el método de medición (TLS o IFT) afectó significativamente a la obtención de las variables dendrométricas DAP y altura total a nivel de árbol individual, se aplicó la prueba no paramétrica de Wilcoxon para datos pareados (dos muestras dependientes) para un nivel de significación estadística $\mathrm{p}<0.05$. La necesidad de implementar una prueba no paramétrica se debió a la comprobación, mediante la prueba de Shapiro-Wilk $(\mathrm{p}<0.05)$, de que las distribuciones de las variables DAP y alturas totales (h) no eran normales.

6. Cálculo de las diferencias (residuos) entre los valores de DAP y h de cada árbol para cada tipo de inventario según las siguientes expresiones: 


$$
\begin{gathered}
\text { Residuo DAP }=\mathrm{DAP}_{\mathrm{TLS}}-\mathrm{DAP}_{\text {tradicional }} \\
\text { Residuo } \mathrm{h}=\mathrm{h}_{\mathrm{TLS}}-\mathrm{h}_{\text {tradicional }} .
\end{gathered}
$$

Dada la naturaleza no normal de la distribución de residuos, se emplearon estimadores robustos como la mediana (Med) y la desviación absoluta con respecto a la mediana (MAD; Mean Absolute Deviation). La mediana es una medida de tendencia central que ofrece la ventaja de ser insensible a la presencia de valores atípicos. Lo mismo se puede decir sobre MAD como estimador de escala o dispersión de los valores entorno a la mediana. El MAD puede estimarse mediante la expresión MAD $=1.4826$ mediana $\left(\mid r_{i}-\right.$ Med $\left.\mid\right)$, donde $r_{i}$ es el valor del residuo i-ésimo (Rousseeuw y Croux, 1993).

Finalmente, al conjunto de datos de los árboles que fueron identificados por sus coordenadas exactas (sistema de referencia WGS84 UTM17S) mediante el TLS, se le aplicó un proceso de verificación y validación para detectar errores en la identificación de los árboles considerando que los inventarios se realizaron con una diferencia de tiempo de entre seis y ocho meses, aproximadamente. Se eliminaron todos los árboles con datos faltantes, fue el caso de aquellos para los que se habían medido las coordenadas (posición), pero no se pudieron extraer los valores de DAP y/o altura en el inventario digital por problemas con la segmentación en 3DForest. En otros casos se eliminaron los que no tenían sus correspondientes homólogos en el inventario tradicional, por estar fuera de la parcela de inventario terrestre de radio $12.62 \mathrm{~m}$, pero sí dentro de la parcela TLS de radio $18 \mathrm{~m}$.

\section{Resultados}

En la tabla 2 se presentan los resultados de la prueba $\mathrm{t}$ Student para muestras independientes aplicada a los promedios muestrales obtenidos para las variables dasométricas seleccionadas (DAP, altura total y radio promedio de copas) y para cada una de las tres plantaciones.

Para la variable DAP, los estadísticos inferenciales de la prueba $t$ indican que no hay evidencia estadística que permita rechazar la hipótesis que supone que la diferencia de medias es significativamente distinta a cero. Por tanto, no se detectaron diferencias estadísticamente significativas entre los dos métodos de inventario aplicados para su estimación. Por otra parte, la tecnología TLS también tiene una elevada precisión en la medición de métricas de los objetos a distancia, cuestión que ha sido confirmada en varias publicaciones al respecto (Mohammed, Majid e Izah, 2018; Maas, Bienert, Scheller y Keane, 2008; Dassot, Constant y Fournier, 2011).

TABLA 2. Estadísticos descriptivos y contraste de medias (t Student) para los valores promedio a nivel de parcela de las variables

\begin{tabular}{|c|c|c|c|c|c|c|c|c|}
\hline \multirow[b]{2}{*}{ Plantación } & \multirow[b]{2}{*}{ Variable } & \multirow[b]{2}{*}{$\mathrm{N}$} & \multicolumn{2}{|c|}{ Inventario tradicional } & \multicolumn{2}{|c|}{ Inventario TLS } & \multirow[b]{2}{*}{ p-valor } & \multirow[b]{2}{*}{ Significancia } \\
\hline & & & M & SE & $\mathrm{M}$ & SE & & \\
\hline \multirow[t]{3}{*}{ Morondava } & $\operatorname{DAP}(m)$ & 28 & 0.081 & 0.003 & 0.083 & 0.003 & 0.5521 & n.s. \\
\hline & $\mathrm{H}(\mathrm{m})$ & 28 & 7.369 & 0.290 & 8.334 & 0.329 & 0.0322 & * \\
\hline & $\mathrm{R}(\mathrm{m})$ & 28 & 1.583 & 0.045 & 1.065 & 0.048 & 0.0001 & * \\
\hline \multirow[t]{3}{*}{ Allteak } & $\mathrm{DAP}(\mathrm{m})$ & 20 & 0.147 & 0.012 & 0.169 & 0.007 & 0.1146 & n.s. \\
\hline & $\mathrm{H}(\mathrm{m})$ & 20 & 13.587 & 1.024 & 16.231 & 0.576 & 0.0317 & * \\
\hline & $\mathrm{R}(\mathrm{m})$ & 20 & 2.157 & 0.137 & 2.135 & 0.092 & 0.8947 & n.s. \\
\hline \multirow[t]{3}{*}{ El Tecal } & $\mathrm{DAP}(\mathrm{m})$ & 8 & 0.126 & 0.010 & 0.131 & 0.008 & 0.4094 & n.s. \\
\hline & $\mathrm{H}(\mathrm{m})$ & 8 & 12.438 & 1.127 & 13.760 & 0.964 & 0.3876 & n.s. \\
\hline & $\mathrm{R}(\mathrm{m})$ & 8 & 1.860 & 0.139 & 1.480 & 0.105 & 0.0468 & * \\
\hline
\end{tabular}
dendrométricas estudiadas según el método de inventario.

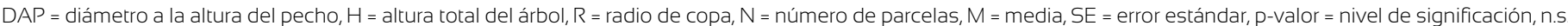
$=$ no significativo, ${ }^{*}=$ significativo $(p<0.05)$. 
En el caso de la variable altura total del árbol, se encontraron diferencias significativas entre el tipo de inventario en las plantaciones de Morondava y Allteak, no siendo significativas en el Tecal. Las diferencias encontradas en las estimaciones de los promedios de la altura media de árbol por parcela en las plantaciones de Morondava y Allteak, con valores significativamente inferiores en el caso del IFT, pueden ser atribuibles a errores de medición debidos a la dificultad para visualizar el ápice del árbol por parte del operador del telémetro láser en las condiciones fenológicas en las que se realizó el inventario tradicional (presencia de hojas). También puede relacionarse con la diferencia de entre seis y ocho meses entre los dos inventarios, lo que implicaría un mayor desarrollo de los árboles en el caso del inventario TLS. Adicionalmente, el inventario TLS se realizó en condiciones de ausencia de hojas, lo que permite obtener una mejor definición del ápice del árbol que en condiciones de follaje denso.

Por último, la variable radio de copa mostró diferencias significativas entre los dos tipos de inventarios en las plantaciones de Morondava y el Tecal, no encontrándose diferencias en el caso de Allteak. Para la variable radio de copa, las diferencias pueden ser también explicadas como consecuencia de la variación en la condición fenológica del árbol. En efecto, la estimación del tamaño de copa tendería a otorgar mayores valores en la estación climática húmeda (árboles con hojas) que en la seca (árboles sin hojas).

En la tabla 3 se muestran los resultados del contraste de promedios por parcela para las variables estudiadas según el tamaño de la parcela TLS (parcela original de 1018 $\mathrm{m}^{2}$ y parcela recortada de $500 \mathrm{~m}^{2}$ ).

La separación de medias no ofreció diferencias significativas $(\mathrm{p}<0.05)$ para ninguna de las variables dendrométricas consideradas (Tabla 2). Este resultado evidencia que el tamaño de parcela circular TLS de radio $12.62 \mathrm{~m}$, equivalente a $500 \mathrm{~m}^{2}$, ofreció unos promedios de DAP, $\mathrm{H}$ y $\mathrm{R}$, estadísticamente similares a los obtenidos a partir de una parcela de radio $18 \mathrm{~m}$ (i.e., el doble de tamaño). Este resultado apunta a la posibilidad de reducir el tamaño de las parcelas de referencia para incrementar la eficiencia del inventario con tecnología TLS.

Tal y como puede observarse en la tabla 4, la prueba $t$ Student no indicó diferencias estadísticamente significativas entre el tiempo medio de trabajo por parcela para llevar a cabo el inventario según los dos métodos testados en las plantaciones de Morondava y Allteak. En el caso del Tecal, la prueba estadística detectó diferencias significativas a un nivel de $\mathrm{p}<0.05$, con una diferencia media a favor del método TLS de 20 minutos. Es importante destacar que el tiempo de medición promedio de una parcela TLS y IFT es de 23 y 18 min, respectivamente. La eficiencia relativa (E) que aparece en la tabla 4, determinada tomando como referencia el método IFT, no mostró valores claramente superiores a la unidad en el caso de la plantación de Morondava, lo que indica que en plantaciones jóvenes el inventario tradicional resulta competitivo con el inventario TLS para la extracción de las variables dendrométricas básicas. Sin embargo, en plantaciones con un mayor desarrollo, como el Tecal y Allteak, la eficiencia del inventario TLS resulta superior a la del IFT, sobre todo si tenemos en cuenta, como ya indicamos anteriormente, el mayor número de árboles que comprende el muestreo TLS.

A escala de árbol se compararon las variables dendrométricas (DAP y H) obtenidas mediante IFT y TLS en los árboles de las tres plantaciones de estudio, con una población final de 1087 árboles. Hay que destacar que el proceso de verificación y validación de datos permitió detectar errores en la identificación de los árboles considerando que los inventarios se realizaron con una diferencia de tiempo de entre seis y ocho meses. Por otra parte, el inventario terrestre se realizó en condiciones fenológicas del cultivo a la salida de lluvias, i.e. árboles con hojas (leaf-on), mientras que el inventario digital se realizó en noviembre, época seca cuando los árboles pierden o ya han perdido sus hojas (leaf-off). 
TABLA 3. Estadísticos descriptivos y contraste de medias (t Student) para los valores promedio a nivel de parcela de las variables dendrométricas estudiadas según la extensión de la parcela TLS (original y recortada).

\begin{tabular}{ccccccccc}
\hline & & \multicolumn{2}{c}{ TLS12 } & \multicolumn{7}{c}{ TLS18 } \\
\hline Plantación & Variable & $\mathrm{N}$ & $\mathrm{M}$ & $\mathrm{SE}$ & $\mathrm{M}$ & $\mathrm{SE}$ & $\mathrm{p}$-valor & Significación \\
\hline Morondava & $\mathrm{DAP}(\mathrm{m})$ & 28 & 0.084 & 0.003 & 0.083 & 0.003 & 0.815 & n.s. \\
& $\mathrm{H}(\mathrm{m})$ & 28 & 8.393 & 0.340 & 8.334 & 0.329 & 0.902 & n.s. \\
& $\mathrm{R}(\mathrm{m})$ & 28 & 1.089 & 0.052 & 1.054 & 0.048 & 0.6234 & n.s. \\
Allteak & $\mathrm{DAP}$ & 20 & 0.178 & 0.006 & 0.169 & 0.007 & 0.349 & n.s. \\
& $\mathrm{H}(\mathrm{m})$ & 20 & 17.108 & 0.551 & 16.231 & 0.576 & 0.2782 & n.s. \\
& $\mathrm{R}(\mathrm{m})$ & 20 & 2.309 & 0.102 & 2.134 & 0.092 & 0.2114 & n.s. \\
& $\mathrm{DAP}(\mathrm{m})$ & 8 & 0.135 & 0.008 & 0.131 & 0.008 & 0.9999 & n.s. \\
& $\mathrm{H}(\mathrm{m})$ & 8 & 14.141 & 0.868 & 13.760 & 0.964 & 0.7732 & n.s. \\
& $\mathrm{R}(\mathrm{m})$ & 8 & 1.590 & 0.103 & 1.480 & 0.105 & 0.4668 & n.s. \\
\hline
\end{tabular}

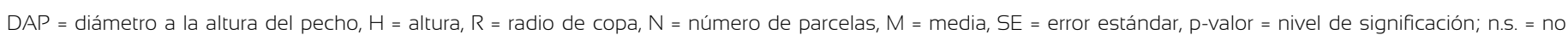
significativa; * = significativa; TLS12 = parcela escáner láser terrestre radio $12.62 \mathrm{~m}$, TLS18 = parcela escáner láser terrestre radio 18 m.

TABLA 4. Eficiencia relativa (E) para la realización del inventario según los métodos inventario forestal tradicional y con escáner láser terrestre.

\begin{tabular}{|c|c|c|c|c|c|c|c|c|c|c|}
\hline Plantación & Variable & $\begin{array}{l}\text { Unidad } \\
\text { medida }\end{array}$ & $N$ & $\begin{array}{c}\mathrm{SE}_{1} \\
\text { (IFT) }\end{array}$ & $\begin{array}{c}\mathrm{SE}_{2} \\
\text { (TLS) }\end{array}$ & $\begin{array}{c}\mathrm{T}_{1} \\
\text { (IFT) }\end{array}$ & $\begin{array}{c}\mathrm{T}_{2} \\
\text { (TLS) }\end{array}$ & p-valor & Sig. & $E$ \\
\hline \multirow[t]{4}{*}{ Morondava } & DAP & $\mathrm{m}$ & 23 & 0.003 & 0.003 & 95 & 94 & - & - & 0.99 \\
\hline & $\mathrm{H}$ & $\mathrm{m}$ & 23 & 0.26 & 0.28 & 95 & 94 & - & - & 1.10 \\
\hline & R & $\mathrm{m}$ & 23 & 0.05 & 0.05 & 95 & 94 & - & - & 0.71 \\
\hline & $\mathrm{T}$ & $\min$ & 23 & - & - & 95 & 94 & 0.940 & n.s & \\
\hline \multirow[t]{4}{*}{ All Teak } & DAP & $\mathrm{m}$ & 18 & 0.01 & 0.01 & 89 & 93 & - & - & 1.97 \\
\hline & $\mathrm{H}$ & $\mathrm{m}$ & 18 & 0.97 & 0.51 & 89 & 93 & - & - & 1.98 \\
\hline & $\mathrm{R}$ & $\mathrm{m}$ & 18 & 0.14 & 0.09 & 89 & 93 & - & - & 1.45 \\
\hline & $\mathrm{T}$ & $\min$ & 18 & - & - & 89 & 93 & 0.612 & n.s & \\
\hline \multirow[t]{4}{*}{ El Tecal } & DAP & $\mathrm{m}$ & 8 & 0.01 & 0.01 & 114 & 94 & - & - & 1.58 \\
\hline & $\mathrm{H}$ & $\mathrm{m}$ & 8 & 1.13 & 0.96 & 114 & 94 & - & - & 1.56 \\
\hline & $\mathrm{R}$ & $\mathrm{m}$ & 8 & 0.14 & 0.11 & 114 & 94 & - & - & 1.27 \\
\hline & T & $\min$ & 8 & - & - & 114 & 94 & 0.037 & * & \\
\hline
\end{tabular}

Para la determinación de la eficiencia se ha tomado como referencia el inventario tradicional (subíndice l en la ecuación l).

$\mathrm{DAP}=$ diámetro a la altura del pecho, $\mathrm{H}=$ altura total del árbol, $\mathrm{R}=$ radio de copa, $\mathrm{m}=$ metros, $\mathrm{N}=$ número de parcelas, $\mathrm{SE}=$ error estándar, $\mathrm{T}$ = tiempo, Sig. = significación, n.S. $=$ no significativo, ${ }^{*}=$ significativo, $\mathrm{E}=$ eficiencia relativa, $\mathrm{TLS}=$ inventario escáner láser terrestre, IFT = inventario forestal tradicional. 
Se pudo evidenciar que el inventario TLS presenta algunas desventajas en los casos de presencia de hojas a la altura de la extracción del DAP (i.e., $1.3 \mathrm{~m}$ ), lo que suele ocurrir, generalmente, en árboles de porte muy pequeño. En este sentido, es recomendable la realización del inventario mediante sensor TLS en condiciones leaf-off, lo que coincide con los meses de noviembre y diciembre en el caso de la Región Costa de Ecuador. Este procedimiento evita, en la medida de lo posible, la presencia de vegetación que limita el rendimiento de los algoritmos de segmentación de la nube de puntos láser y el ajuste de circunferencias para la determinación de diámetros de tronco (Aguilar et al., 2019a).

Los resultados de la comparación de las dos variables dendrométricas extraídas a nivel de árbol se detallan en la tabla 5. En la plantación de Morondava, la prueba estadística de Wilcoxon evidenció diferencias significativas en las mediciones de altura y diámetro de los árboles entre los dos métodos de inventario testados, al igual que ocurrió en la plantación de Allteak. En el caso de El Tecal solo se detectaron diferencias significativas en la medición del DAP, no resultando significativas las diferencias en la medición de la altura del árbol. Un análisis detallado de los residuos de los datos pareados para DAP y $\mathrm{H}$ se muestra en la tabla 6 .

En el predio de Morondava la media de los residuos del DAP (Tabla 6) de los 644 árboles comparados es de 0.34 $\mathrm{cm}$, mientras que la media de los residuos de la altura total es de $0.99 \mathrm{~m}$, mostrando además desviaciones estándar pequeñas. Se observa cómo los estimadores robustos como la mediana o el MAD arrojan valores ligeramente inferiores (0.32 cm y $0.97 \mathrm{~m}$, respectivamente), apuntando, como se mencionaba anteriormente, a una naturaleza no normal de la distribución de los residuos debido a la presencia de valores atípicos.

Los resultados obtenidos indican que la extracción del DAP y $\mathrm{H}$ a partir de nubes de puntos TLS ha sobreestimado ligeramente las medidas de ambas variables. En el caso de la variable $\mathrm{H}$ la sobreestimación alcanza valores medios de casi $1 \mathrm{~m}$, lo que resulta excesivo, dado el pequeño tamaño y prácticamente ausencia de hojas en los árboles de esta plantación. En efecto, en el momento del inventario con el sensor TLS los árboles de todas las parcelas de referencia en Morondava no presentaban prácticamente hojas, lo que aseguraba una precisa y fiable extracción de parámetros geométricos a partir de la nube de puntos TLS.

TABLA 5. Prueba no paramétrica de Wilcoxon para datos pareados (variables dependientes) aplicado a variables dendrométricas, a escala de árbol individual, extraídas mediante inventario tradicional e inventario TLS.

\begin{tabular}{cccccccccc}
\hline & \multicolumn{7}{c}{ IFT } & \multicolumn{7}{c}{ TLS } \\
\hline Plantación & Variable & N & M & SE & M & SE & P-valor & Significación \\
\hline \multirow{2}{*}{ Morondava } & DAP $(\mathrm{m})$ & 644 & 0.090 & 0.017 & 0.093 & 0.016 & $<0.0001$ & $*$ \\
& $\mathrm{H}(\mathrm{m})$ & 644 & 8.19 & 1.66 & 9.18 & 1.58 & $<0.0001$ & $*$ \\
& $\mathrm{DAP}(\mathrm{m})$ & 209 & 0.194 & 0.044 & 0.189 & 0.038 & $<0.0001$ & $*$ \\
Allteak & $\mathrm{H}(\mathrm{m})$ & 209 & 18.67 & 4.06 & 18.04 & 2.93 & 0.00061 & $*$ \\
& $\mathrm{DAP}(\mathrm{m})$ & 234 & 0.149 & 0.038 & 0.141 & 0.038 & $<0.0001$ & $*$ \\
El Tecal & $\mathrm{H}(\mathrm{m})$ & 234 & 14.71 & 3.39 & 14.82 & 3.08 & 0.0612 & n.s. \\
\hline
\end{tabular}

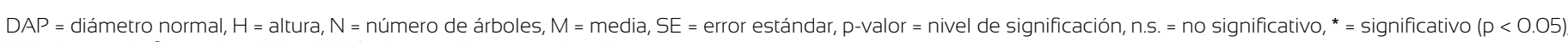
IFT = inventario forestal tradicional, TLS = inventario con escáner láser terrestre. 
TABLA 6. Media, desviación estándar (SD), mediana y desviación absoluta de la media (MAD; Mean Absolute Deviation) de los residuos (Medida $a_{\text {TLS }}$ - Medidatradicional) de DAP (cm) y altura total H (m) de los árboles de muestra.

\begin{tabular}{lcccccc}
\hline & \multicolumn{2}{c}{ Morondava, 644 árboles } & \multicolumn{2}{c}{ Alteak, 209 árboles } & \multicolumn{2}{c}{ El Tecal, 234 árboles } \\
\hline & DAP $(\mathrm{cm})$ & $\mathrm{H}(\mathrm{m})$ & $\mathrm{DAP}(\mathrm{cm})$ & $\mathrm{H}(\mathrm{m})$ & DAP $(\mathrm{cm})$ & $\mathrm{H}(\mathrm{m})$ \\
\hline Media & 0.34 & 0.99 & -0.51 & -0.62 & -0.79 & 0.11 \\
SD & 0.86 & 0.88 & 1.97 & 2.29 & 1.10 & 2.15 \\
Mediana & 0.32 & 0.97 & -0.40 & -0.40 & -0.78 & 0.26 \\
MAD & 0.76 & 0.70 & 1.04 & 1.84 & 0.90 & 1.63 \\
\hline
\end{tabular}

Una explicación razonable a esta sobreestimación estaría relacionada con el hecho de la diferencia de entre seis y ocho meses entre ambas campañas. Teniendo en cuenta que se trata de una plantación muy joven (media de 2.9 años en el momento del inventario TLS), se puede asumir que los árboles de teca de Morondava han crecido más que los árboles de las otras dos plantaciones. Este razonamiento podría ser aplicado tanto a la sobreestimación del DAP como, y especialmente, a la sobreestimación de la altura total.

En el caso del predio Allteak, la media de los residuos de DAP, calculada para 209 árboles, tomó un valor de -0.51 $\mathrm{cm}$, mientras que la media de los residuos de la altura total resultó ser de $-0.62 \mathrm{~m}$. Los valores de mediana y MAD fueron significativamente inferiores en valor absoluto ($0.40 \mathrm{~cm}$ y $-0.40 \mathrm{~m}$, respectivamente), lo que apunta a una clara presencia de valores atípicos en la muestra. En cualquier caso, los valores registrados implican una subestimación en la extracción de dichos parámetros a partir del inventario TLS.

La subestimación del DAP podría deberse al redondeo al alza aplicado para anotar su valor en el caso de la campaña terrestre. En cuanto a la altura total, la ligera, pero estadísticamente significativa, subestimación se debió a que los inventarios tradicional y TLS se realizaron en dos momentos fenológicos diferentes (leaf-on y leaf-off, respectivamente). En efecto, la diferencia en la medida podría deberse al efecto distorsionador del follaje situado en el ápice de cada árbol. Téngase en cuenta la gran dificultad para tomar la altura exacta de los árboles en la estación leaf-on a partir de las medidas realizadas con un hipsómetro láser (Trimble Laser Ace 1000 ${ }^{\mathrm{TM}}$ ).

Finalmente, en la plantación de El Tecal también se observó una subestimación del DAP en el caso del inventario TLS, con un valor residual medio de $-0.79 \mathrm{~cm}$ (mediana de $-0.78 \mathrm{~cm}$ ), lo que puede explicarse siguiendo el razonamiento aplicado en el caso de Allteak. En lo que respecta la altura total, el residuo medio calculado para 234 árboles no refleja diferencias significativas entre las medidas realizadas en la campaña terrestre y en la campaña TLS, tomando un valor medio de tan solo $0.11 \mathrm{~m}$.

\section{DISCUSIÓN}

Los resultados obtenidos permiten afirmar que los dos métodos de inventario testados son suficientemente precisos para la medición de las variables dendrométricas estudiadas. Las diferencias observadas entre los resultados ofrecidos por ambos métodos pueden ser atribuibles a los procedimientos instrumentales de medición en las parcelas del inventario tradicional, al tiempo transcurrido entre ambos inventarios y a las diferencias en el estado fenológico (Dassot et al., 2011; Liang, Kankare, Yu, Hyyppa y Holopainen, 2014).

En el caso de la variable altura total, donde se utilizó el hipsómetro láser en el caso de IFT, se detectaron problemas como consecuencia de la dificultad en visualizar el ápice del árbol debido a la presencia de un denso follaje (condiciones leaf-on). Hay que tener en cuenta, además, la diferencia de entre seis y ocho meses entre los dos inventarios, que explicaría la diferencia de altura total entre el inventario TLS (muestreo más reciente) y el IFT 
(muestreo más antiguo) en el caso de la plantación de Morondava, con árboles de teca en pleno crecimiento (Saarinen et al., 2017; Maas et al., 2008). En el caso de la variable radio de corona, las diferencias registradas entre ambos métodos de inventario pueden ser también atribuibles al cambio de condición fenológica de la plantación.

Las pruebas estadísticas no detectaron diferencias significativas entre el método de medición por TLS y el método de medición tradicional cuando se aplicó a nivel de parcela de referencia (valores promedio), con excepción de las variables altura total y radio de copa en algunos casos. En el caso de Morondava, las diferencias en altura se atribuyen al crecimiento de los árboles entre los dos inventarios. En el caso del radio de corona se obtienen diferencias debido a diferentes estados fenológicos de la plantación durante los inventarios comparados. Los resultados obtenidos son muy similares a los publicados por Mohammed et al. (2018), donde reportaron que las diferencias entre el inventario TLS y el IFT no son estadísticamente significativas. Resultados similares fueron obtenidos por Rodríguez y Klaribel (2018), en Ecuador, evaluando tecnologías TLS y IFT. Ellos llegaron a la conclusión de que, en términos estadísticos, los valores obtenidos por medición en parcelas de campo realizados por el método convencional son similares a los valores obtenidos por la medición realizada mediante TLS.

Por otra parte, Uzquiano y Bravo (2017) resaltan que uno de los problemas de la técnica TLS es que el procesamiento de datos necesita mucho tiempo, criterio que se comparte en este estudio. No se encontró evidencia de una considerable mayor eficiencia del método TLS para obtener las variables dendrométricas básicas. En efecto, se trata de una tecnología emergente en aplicaciones forestales que requiriere de un elevado nivel técnico y habilidad en el uso de software complejo para la extracción de métricas. Algunos procedimientos pueden considerarse semiautomáticos, mientras que otros requieren de una participación continua de un operario capacitado. Esto hace que la aplicación de la tecnología TLS en el inventario forestal para la obtención de variables dendrométicas básicas sea actualmente una herramienta de difícil introducción en este medio. Sin embargo, hay que destacar que el potencial de la tecnología TLS en el ámbito del inventario forestal no solo se limita a la extracción de variables dendrométricas simples como DAP o altura total, sino que puede considerarse como un método de medición eficiente y no destructivo que está produciendo un verdadero replanteamiento de los estudios forestales (Suraj Reddy et al., 2018; Du, Lindenbergh, Ledoux, Stoter y Nan, 2019), siendo empleado para el soporte en la generación de modelos alométricos (Lau et al., 2019; Kankare et al., 2013; Calders et al., 2015), la elaboración de ecuaciones de la forma del tronco (Liang et al., 2014), e incluso para la obtención de una completa descripción en 3D de la arquitectura del árbol (Raumonen et al., 2013; Hackenberg, Spiecker, Calders, Disney y Raumonen, 2015; Delagrange, Jauvin y Rochon, 2014).

$\mathrm{El}$ inventario tradicional terrestre presenta problemas relacionados con la ubicación espacial de cada árbol, especialmente cuando se realiza en condiciones leaf-on. En este caso, las técnicas GPS-RTK de precisión para determinar la posición espacial de cada árbol en la parcela de muestreo se ven limitadas por la interferencia en la recepción de la señal producida por las copas de los árboles. Estos procedimientos de medición en campo son realizados por diferentes operadores, lo que puede inducir errores de medición y de registro de datos. Por último, todos los procedimientos, al ser ejecutados por personal con diferentes grados de capacitación y experiencia, introducen errores que en la reiteración del inventario hacen difícil su actualización a los efectos del monitoreo del crecimiento de la plantación (Liang et al., 2016).

\section{CONCLUSIONES}

A escala de sitio de muestreo, la comparación de los métodos de inventario TLS y IFT no ofreció diferencias significativas en los valores promedio por parcela de referencia de la variable dendrométrica DAP en plantaciones de teca de la Región Costa de Ecuador. La diferencia en los valores promedio de la variable altura total, con unos valores significativamente mayores en el caso del 
inventario TLS, pudieron deberse al crecimiento de los árboles durante el intervalo transcurrido entre ambos inventarios. Adicionalmente, hay que tener en cuenta los errores de medición de alturas mediante hipsómetro láser (método tradicional) debido a la ejecución del inventario en una condición fenológica "leaf-on" que produjo la dificultad en la localización del ápice del árbol en numerosas ocasiones. Los valores de radio de corona significativamente mayores en el IFT pueden atribuirse a que el inventario TLS se llevó a cabo en condiciones "leafoff', extrayéndose un radio de corona, o contorno aparente de la nube de puntos, más reducido que en condiciones "leaf-on".

La evaluación del efecto de la reducción del tamaño de parcela de medición TLS en la precisión de la estimación de las variables dendrométricas no mostró diferencias significativas, siendo este un resultado de interés para reducir los tiempos de trabajo en la extracción de métricas dendrométricas al implicar una disminución significativa del número de árboles incluidos en la unidad de muestreo.

No se encontraron diferencias significativas entre los tiempos de trabajo de campo para el levantamiento de parcelas según el método de inventario tradicional y parcelas TLS. La eficiencia relativa calculada demostró ligera superioridad del método de inventario TLS sobre el IFT, lo que apunta a la necesidad de incrementar la investigación en la línea de hacer más eficientes los métodos de procesamiento de nubes de puntos para una extracción automática de métricas.

La comparación de métodos de inventario a escala de árboles puso en relieve la dificultad en la recepción de la señal GPS en zonas de denso follaje (condiciones leaf-on) en el momento de realizar el inventario tradicional, lo que dificultó en gran medida la determinación exacta de la ubicación planimétrica espacial de los árboles. En el caso de esta investigación, este problema ha sido solventado con éxito adoptando la metodología propuesta en este estudio y realizando el inventario digital en condiciones leaf-off. Estos resultados recomiendan la realización de inventarios mediante sensores remotos terrestres en la temporada seca de la Región Costa de Ecuador, momento en el que la mayoría de las plantaciones de teca han perdido sus hojas. Este tipo de inventarios realizados en condiciones leaf-off pueden permitir un efectivo y preciso monitoreo del crecimiento de las plantaciones, minimizando los posibles errores del operario.

La comparación de los resultados de los datos del inventario forestal TLS con los obtenidos mediante la metodología tradicional arroja resultados muy aceptables. Uno de los principales inconvenientes encontrados en la realización del experimento con el inventario mediante el sensor TLS está relacionada con la presencia de hojas a la altura de la extracción del diámetro normal, por lo que se recomienda que se realice en condiciones leaf-off. Como ventaja adicional, las mediciones se realizan con un solo equipo de alta precisión para extraer todas las métricas posibles del árbol sin la subjetividad que puede introducir el operador de los instrumentos de medición terrestre.

Por último, hay que destacar que el potencial de la tecnología TLS en el ámbito del inventario forestal no solo se limita a la extracción de variables dendrométricas simples como DAP o altura total, sino que puede emplearse en otras muchas aplicaciones como la generación de modelos alométricos, el desarrollo de ecuaciones de la forma del tronco o la obtención de una completa descripción 3D de la arquitectura del árbol.

\section{RECONOCIMIENTOS}

Los autores desean agradecer a Emily Polo, Álvaro C. Díaz Chacho y Javier A. Mora Espinoza (Universidad Católica de Santiago de Guayaquil) por su valiosa ayuda en el trabajo de campo para realizar el inventario forestal entre los meses de marzo a junio de 2018. Finalmente, agradecemos a los revisores anónimos por sus valiosos comentarios y sugerencias. Esta investigación fue financiada por el proyecto "Evaluación de tecnologías de detección remota para la estimación de biomasa de teca en la Región Costa de Ecuador" (Sistema de Investigación y Desarrollo del Universidad Católica de Santiago de Guayaquil, Ecuador). Este trabajo también forma parte de las líneas generales de investigación del proyecto "Enabling interdisciplinary COllaboration to Foster Mediterranean foREST 
sustainable management and Socio-ECOnomic valuation (ECO2-FOREST)" (Proyecto Retos Junta de Andalucía P18-RT-2327, España) y del Campus Agroalimentario de Excelencia Internacional ceiA3, España.

\section{REFERENCIAS}

Abegg, M., Kükenbrink, D., Zell, J., Schaepman, M., \& Morsdorf, F. (2017). Terrestrial láser scanning for forest Inventories-Tree diameter distribution and scanner location impact on occlusion. Forests, 8(6), 184. doi: 10.3390/f8060184

Aguilar, F. J., Nemmaoui, A., Peñalver, A., Rivas, J. R., \& Aguilar, M. A. (2019a). Developing Allometric Equations for Teak Plantations Located in the Coastal Region of Ecuador from Terrestrial Laser Scanning Data. Forests, 10(12), 1050. doi:10.3390/f10121050

Aguilar, F. J., Rivas, J. R., Nemmaoui, A., Peñalver, A., \& Aguilar, M. A. (2019b). UAV-Based Digital Terrain Model Generation under Leaf-Off Conditions to Support Teak Plantations Inventories in Tropical Dry Forests. A Case of the Coastal Region of Ecuador. Sensors, Forests, 19(8), 1934. doi:10.3390/s19081934

ARCGIS (Versión 10.3). (2014). [Create and Design Maps and 3D Scenes]. ESRI. https://desktop.arcgis.com/en/

Bahamondez, C., Lorenz, M., Mery, G., \& Varjo, J. (2005). Evaluación de los recursos forestales ante necesidades cambiantes de información. Second Latin American IUFRO Congress, La Serena, Chile, 23-27 October 2006. International Union of Forest Research. La Serena: Chile.

Bare, B. B. (June 13-17, 2003). Opening remarks and welcome to the first international precision forestry symposium. Second International Precision Forestry Symposium, Seatle, USA. University of Washington: USA.

Calders, K., Newnham, G., Burt, A., Murphy, S., Raumonen, P., Herold, M., Culvenor, D., Avitabile, V., Disney, M., Armston, J., \& Kaasalainen, M. (2015). Nondestructive estimates of aboveground biomass using terrestrial laser scanning. Methods in Ecology and Evolution, 6(2), 198-208. doi: 10.1111/2041-210X.12301

Dassot, M., Constant, T., \& Fournier, M. (2011). The use of terrestrial LiDAR technology in forest science: application fields, benefits and challenges. Annals of Forest Science, 68, 959-974. doi: 10.1007/s13595-011-0102-2

Delagrange, S., Jauvin, C., \& Rochon, P. (2014). Pype'Tree: A Tool for Reconstructing Tree Perennial Tissues from Point Clouds. Sensors, 14(3), 4271-4289. doi: 10.3390/s140304271
Du, S., Lindenbergh, R., Ledoux, H., Stoter, J., \& Nan, L. (2019). AdTree: Accurate, Detailed, and Automatic Modelling of LaserScanned Trees. Remote Sensing, 11, 2074.

Flores Velasteguí, T., Cabezas Guerrero, F., \& Crespo Gutiérrez, R. (2010) Plagas y enfermedades en plantaciones de TecaTeca (Tectona grandis L.F.) en la zona de Balzar, provincia de Guayas. Ciencia y Tecnologia, 3, 15-22.

Hackenberg, J., Spiecker, H., Calders, K., Disney, M., \& Raumonen, P. (2015). SimpleTree-An Efficient Open Source Tool to Build Tree Models from TLS Clouds. Forests, 6(11), 4245-4294. doi: $10.3390 /$ f 6114245

Holdridge, L. R. (1982). Ecología basada en zonas de vida, serie de Libros y Materiales Educativos. San José, Costa Rica: Instituto Interamericano de Cooperación para la Agricultura (IICA).

INFOSTAT (2010). Software para análisis estadístico de aplicación general. Universidad Nacional de Córdoba (FCA-UNC). Recuperado de https://www.infostat.com.ar/

Kankare, V., Holopainen, M., Vastaranta, M., Puttonen, E., Yu, X., Hyyppä, J., Vaaja, M., Hyyppä, H., \& Alho, P. (2013). Individual tree biomass estimation using terrestrial laser scanning. ISPRS Journal of Photogrammetry and Remote Sensing, 75, 64-75. doi: 10.1016/j.isprsjprs.2012.10.003

Kershaw, J. A., Ducey, M. J., Beers, T. W., \& Husch, B. (2017). Integrating remote sensing in forest inventory. En J. W. \& Sons (Ed.), Forest Mensuration (5th ed., pp. 429-454). Chichester, UK: John Wiley y Sons, Ltd. doi: 10.1002/9781118902028.ch13

Lau, A., Calders, K., Bartholomeus, H., Martius, C., Raumonen, P., Herold, M., Vicari, M., Sukhdeo, H., Singh, J., \& Goodman, R. (2019). Tree Biomass Equations from Terrestrial LiDAR: A Case Study in Guyana. Forests, 10(6), 527. doi: 10.3390/f10060527

Liang, X., Kankare, V., Yu, X., Hyyppä, J., \& Holopainen, M. (2014). Automated Stem Curve Measurement Using Terrestrial Laser Scanning. IEEE Transactions in Geosciences and Remote Sensing, 52(3), 1739-1748. doi: 10.1109/TGRS.2013.2253783

Liang, X., Kankare, V., Hyyppä, J., Wang, Y., Kukko, A., Haggrén, H., Yu, X., Kaartinen, H., Jaakkola, A., \& Guan, F. (2016). Terrestrial laser scanning in forest inventories. ISPRS Journal of Photogrammetry and Remote Sensing, 115, 63-77. doi: 10.1016/j.isprsjprs.2016.01.006

Maas, H. G., Bienert, A., Scheller, S., \& Keane, E. (2008). Automatic forest inventory parameter determination from terrestrial laser scanner data. International Journal of Remote Sensing, 29, 1579-1593. doi: 10.1080/01431160701736406

Mohammed, H. I., Majid, Z., \& Izah, L. N. (2018, June). Terrestrial laser scanning for tree parameters inventory. IOP Conference Series: Earth and Environmental Science, 169(1), 012096. 
Porras Díaz, H., Cáceres Jiménez, J. J., \& Gallo Lancheros, E. O. (2014). Three-dimensional urban models generated from point clouds of a terrestrial laser scanner. Tecnura, 18(41), 134-153.

Ramos, L., Marchamalo, M., Rejas, J. G., \& Martínez, R. (2015). Aplicación del Láser Escáner Terrestre (TLS) a la modelización de estructuras: precisión, exactitud y diseño de la adquisición de datos en casos reales. Informes de la Construcción, 67(538), e074. doi: $10.3989 /$ ic. 13.103

Raumonen, P., Kaasalainen, M., Åkerblom, M., Kaasalainen, S., Kaartinen, H., Vastaranta, M., Holopainen, M., Disney, M., \& Lewis, P. (2013). Fast Automatic Precision Tree Models from Terrestrial Laser Scanner Data. Remote Sensing, 5(2), 491-520. doi: $10.3390 /$ rs5020491

Rodríguez, B., \& Klaribel, J. (2018). Análisis dasométrico aplicando tecnología escáner láser terrestre y técnicas convencionales para la estimación de biomasa aérea en el bosque la Armenia. Tesis de Grado, Universidad de las Fuerzas Armadas ESPE, Carrera de Ingeniería Geográfica y del Medio Ambiente, Quito, Ecuador.

Rousseeuw, P. J., \& Croux, C. (1993). Alternatives to the Median Absolute Deviation. Journal of American Statistical Association, 88(424), 1273-1283. doi: 10.1080/01621459.1993.10476408

Saarinen, N., Kankare, V., Vastaranta, M., Luoma, V., Pyörälä, J., Tanhuanpää, T., Liang, X., Kaartinen, H., Kukko, A., Jaakkola, A., Yu, X., Holopainen, M. \& Hyppä, J. (2017). Feasibility of Terrestrial laser scanning for collecting stem volume information from single trees. ISPRS Journal of Photogrammetry and Remote Sensing, 123, 140-158. doi: 10.1016/j.isprsjprs.2016.11.012

Scene (2017). Procesa y administra datos de escaneo para aplicaciones de documentación 3D. Versión 7.1. FARO. Recuperado de https://knowledge.faro.com/Software/FARO_SCENE

Suraj Reddy, R., Rakesh, A., Jha, C.S., Rajan, K.S. (2018). Automatic Estimation of Tree Stem Attributes Using Terrestrial Laser Scanning in Central Indian Dry Deciduous Forests. Current Science, 114(1), 201-206.
Trochta, J., Krůček, M., Vrška, T., \& Král, K. (2017). 3D Forest: An application for descriptions of three-dimensional forest structures using terrestrial LiDAR. PLOS ONE, 12(5), 1-17. doi: 10.1371/journal.pone.0176871

Uzquiano, S., \& Bravo, F. (2017). Procesado de datos a través del Láser Escáner Terrestre (TLS): desarrollo de una nueva metodología aplicada a bosques mixtos. Cuadernos de la Sociedad Española de Ciencias Forestales, 43, 225-238.

White, J., Coops, N. C., Wulder, M. A., Vastaranta, M., Hilker, T., \& Tompalski, P. (2016). Remote Sensing Technologies for Enhancing Forest Inventories: A Review. Canadian Journal of Remote Sensing, 42(5), 619-641. doi: 10.1080/07038992.2016.1207484

3D Forest (2016). 3D Forest Ver. v051. Sofware para el análisis de datos TLS. Recuperado de https://www.3dforest.eu/

Manuscrito recibido el 27 de febrero de 2020

Aceptado el 22 de junio de 2020

Publicado el 9 de julio de 2021

Este documento se debe citar como:

Peñalver Romeo, A., Aguilar Torres. F. J., Nemmaoui, A., Rivas Barzola, J. R., Triana Tomalá, A. A., Aguilar Torres, M. A., \& Llanderal Quiroz, A. (2021). Precisión y eficiencia del inventario de plantaciones de teca en Ecuador mediante escáner láser terrestre. Madera y Bosques, 27(1), e2712097. doi: 10.21829/myb.2021.2712097

Madera y Bosques por Instituto de Ecología, A.C. se distribuye bajo una Licencia Creative Commons Atribución-NoComercialCompartirlgual 4.0 Internacional. 